Urologe 2009 · 48:471-472

DOI 10.1007/s00120-009-1973-9

Online publiziert: 11. Mai 2009

(c) Springer Medizin Verlag 2009

\author{
D. Schultz-Lampel \\ Kontinenzzentrum Südwest, Schwarzwald-Baar-Klinikum, Villingen-Schwenningen
}

\title{
Rekonstruktive Beckenchirurgie bei Harnbelastungsinkontinenz und Deszensus der Frau
}

An einer Harninkontinenz leiden 20-50\% aller Frauen. Ebenso viele haben das Risiko, im Laufe ihres Lebens einen Genitaldeszensus zu entwickeln, der nicht nur zu Senkungsbeschwerden, sondern auch zu Inkontinenz oder Blasenentleerungsstörungen sowie zu Stuhlgangsbeschwerden führen kann. Neue Erkenntnisse zum Pathomechanismus der Harninkontinenz und des Deszensus haben dazu geführt, den Beckenboden und die Urogenitalorgane der Frau als komplexe, sich gegenseitig beeinflussende Strukturen anzusehen.

Die rekonstruktiven operativen Verfahren bei Harnbelastungsinkontinenz und Deszensus der Frau sind Leitthema dieses Heftes, das von Mitgliedern des Arbeitskreises „Urologische Funktionsdiagnostik und Urologie der Frau" der Akademie der Deutschen Gesellschaft für Urologie zusammengestellt wurde.

Die Indikation zu einem beckenchirurgischen Eingriff wird in der Regel nach Ausschöpfung und bei Misserfolg konservativer Therapieoptionen gestellt. Die postoperativen Erfolgs- und Zufriedenheitsraten hängen dabei wesentlich von der Patientenselektion und damit von der richtigen Indikationsstellung und somit von der exakten präoperativen Diagnostik ab.

Als Einleitung zum operativen Thema, gibt Daniela Schultz-Lampel (Direktorin des Kontinenzzentrum Südwest in Villingen-Schwenningen und Vorsitzende des Arbeitskreises) einen Überblick über die leitlinienkonforme diagnostischen Empfehlungen und die sich daraus ableitenden therapeutischen Empfehlungen, wie sie aktuell in dem Konsensus der interdisziplinären Leitliniensitzungen der Deutschen Gesellschaft für Gynäkologie und
Geburtshilfe und der Deutschen Gesellschaft für Urologie festgelegt wurden.

Über 90\% der Patientinnen bei Harnbelastungsinkontinenz werden heute mit einem spannungsfreien Band operiert. Stephan Bross (Direktor der Urologischen Klinik in Bruchsal) und Axel Haferkamp (Leitender Oberarzt der Urologischen Klinik der Universitätsklinik in Heidelberg) erörtern in ihrem Beitrag die Vorund Nachteile des retropubischen Prinzip (TVT) und des transobturatorischen $\mathrm{Zu}$ gangs (Prinzip TOT) und versuchen Differentialindikationen für die einzelnen Zugangswege nach dem aktuellen Stand der Literatur abzuleiten.

Obwohl die spannungsfreien Bänder der mittleren Harnröhre die Operationstechniken der Belastungsinkontinenz revolutioniert haben, bleiben noch einige Indikationen für die ehemalige Standardtherapie der Burch-Kolposuspension oder der Faszienzügelplastik. Mark Goepel (Direktor der Urologischen Klinik in Velbert) und Stephan Bross beschreiben in ihrem Beitrag den aktuellen Stellenwert dieser zunehmend verdrängten Verfahren.

In der Vergangenheit war die operative Korrektur eines Deszensus oder Prolaps Domäne der Gynäkologie. In den letzten Jahren werden diese Eingriffe zunehmend auch von Urologen durchgeführt. M. Hamann und Christoph Seif von der Urologischen Universitätsklinik in Kiel, stellen die gültigen Therapieleitlinien, welche Operationsverfahren für welche Form des Deszensus geeignet sind, gemäß den oben bereits erwähnten aktuellen interdisziplinären Leitlinien dar.

Die scheinbar so einfach und minimal-invasiv durchzuführenden Bandund Netzimplantation hat zu einer star- 
ken Ausweitung und teilweise auch unkritischen Stellung der Operationsindikationen geführt. Folge sind teilweise schwerwiegende und oft nur schwer zu korrigierende Komplikationen, deren Häufigkeit und Ausmaß teilweise stark unterschätzt werden. Christian Hampel (Leitender Oberarzt der Urologischen Klinik der Universitätsklinik in Mainz) gibt einen exzellenten Einblick in das große Repertoire der Patienten, die sich mit derartigen Komplikationen am Kontinenzzentrum in Mainz vorstellten und zeigt die spezifischen Patientenprofile, deren Risikofaktoren, sowie das anhand der komplexen Diagnostik abgeleiteten Komplikationsmangement.

Abschließend geben Frau Joanne Nyarangi-Dix und Markus Hohenfellner (Direktor der Urologischen Klinik der Universitätsklinik in Heidelberg) einen zusammenfassenden Überblick über die aktuellen Behandlungskonzepte der rekonstruktiven Beckenbodenchirurgie und die Ausblicke in die Zukunft, bei der durch Weiterentwicklung neuer Verfahren und Materialien Erfolgs- und Zufriedenheitsraten erhöht, sowie die Rezidivund Komplikationsraten reduziert werden sollen.

Gerade bei der Beckenbodenchirurgie der Frau ist eine interdisziplinäre Betrachtungsweise, bei der Urologen, Gynäkologen und Chirurgen zusammenwirken, für eine erfolgreiche Behandlung unerlässlich.

Die Etablierung interdisziplinärer Kontinenz- und Beckenbodenzentren, mit ausgewiesenen Experten auf dem Sektor der Diagnostik und Therapie, ist eine Möglichkeit durch hohe Fallzahlen und operative Spezialisierung dem Ziel der optimalen Patientenversorgung näher zu kommen.

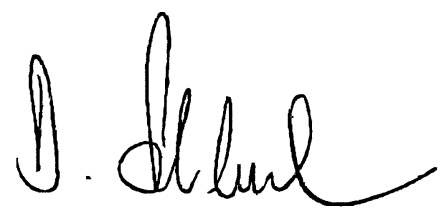

D. Schultz-Lampel

\section{Korrespondenzadresse \\ Prof. Dr. D. Schultz-Lampel Kontinenzzentrum Südwest, Schwarzwald-Baar-Klinikum, Röntgenstraße 20, 78054 Villingen-Schwenningen ksw@sbk-vs.de}

\section{Exklusiv für Abonnenten und Gesellschaftsmitglieder :}

\section{Nutzen Sie das Online-Archiv von Der Urologe}

Ihre Vorteile:

- Komfortable und schnelle Recherche nach Themen, Autoren, Suchbegriffen

— Ob unterwegs oder am eigenen PC: Zugriff überall und jederzeit

— Online First: Lesen Sie die aktuellsten Beiträge schon vor Erscheinen des gedruckten Heftes online

Registrieren Sie sich jetzt unter

www.DerUrologe.de

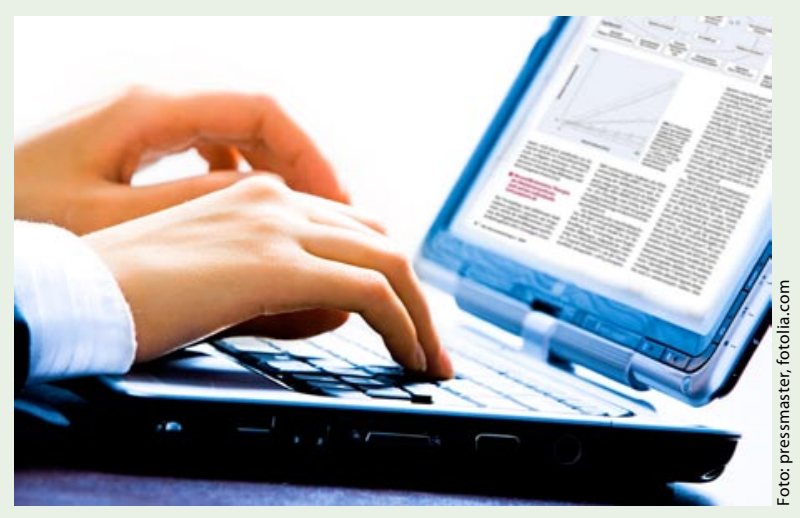

Dieser Online-Service steht auch Mitgliedern der folgenden Fachgesellschaften zur Verfügung:

- Deutsche Gesellschaft für Urologie e.V.

- Berufsverband der Deutschen Urologen e.V. 